\title{
Unsteady flow of a dusty fluid between two parallel plates bounded above by porous medium
}

\author{
Parul Saxena*, Manju Agarwal \\ Department of Mathematics and Astronomy, Lucknow University, Lucknow, INDIA \\ *Corresponding Author: email: pulsxn@gmail.com, Tel. +927897951521 (Parul Saxena), Tel. +929451723146 (Manju Agarwal)
}

\begin{abstract}
The purpose of the present paper is to study an unsteady laminar flow of an incompressible conducting viscous dusty fluid between two infinitely non-conducting parallel plates, where above plate is bounded by porous medium. The flow is influenced by pulsatile pressure gradient, uniform magnetic field which is applied perpendicular to the plates and due to oscillations of the plates under the effect of porosity of porous medium. The governing systems of partial differential equations are solved using finite difference scheme. The effect of permeability of porous medium on the motion in the fluid phase and in the dust phase has been discussed. The effect of magnetic field on the velocity profiles of both fluid and dust phase have been investigated.
\end{abstract}

Keywords: Dusty Fluid, pulsatile pressure gradient, Porous medium, Oscillating plates.

DOI: http://dx.doi.org/10.4314/ijest.v6i1.3

\section{Introduction}

The unsteady flow and heat transfer of a dusty fluid has a wide range of applications in air conditioning, refrigeration, chemical processing, pumps and nuclear reactors. Datta et al. (1993) obtained the solution of unsteady heat transfer to pulsatile flow of a dusty viscous incompressible fluid in a channel. Attia (2006) investigated an unsteady MHD Coutte flow and heat transfer of dusty fluid. Gireesha et al. (2009) obtained the analytical solutions for velocity field using variable separable method for an unsteady flow of dusty fluid through rectangular channel under the influence of pulsatile pressure gradient. Chen (2004) investigated the effect of combined heat and mass transfer on MHD free convection from a vertical surface with the ohmic heating and viscous dissipation. Unsteady hydromagnetic flow and heat transfer from a non-isothermal streching sheet immersed in porous medium was discussed by Chamka (1998). The MHD flow of a dusty viscous conducting fluid between two parallel plates has been investigated by Sreeharireddy et al. (2009). MHD flow and heat transfer of dusty fluid between two parallel plates with variable physical properties have been studied by Attia and Kotb (1996). The results are investigated by varying the parameters linked to the magnetic field and heat transfer and plotting them graphically.

Prasad and Ramacharyulu (1979) initiated the solution of an unsteady flow of a dusty incompressible fluid between two parallel plates under varying impulsive pressure gradient. Unsteady hydromagnetic flow of a dusty viscous fluid between two oscillating plates were investigated by Debnath and Ghosh (1989). Elbashbeshy and Bazid (2004) investigated the heat transfer in a porous medium over a stretching sheet with internal generation and suction or injection. Ezzat et al. (2010) investigated the hydromagnetic flow of a dusty fluid through a porous medium using space approach.

The present investigation deals with the study of an unsteady flow of viscous dusty fluid between two oscillating plates bounded above by porous medium. The flow is due to pulsatile pressure gradient and the uniform magnetic field. The shape of dust particles are spherical in nature and uniformly distributed throughout the fluid. The numerical investigation for the velocities profiles have been done using finite difference method. The effect of porous parameter, magnetic parameter on the fluid and dust particles is discussed. 


\section{Formulation of the Problem}

An unsteady laminar flow of an incompressible, viscous dusty fluid between two plates separated by a distance h is considered. Throughout the flow analysis the following assumptions are made,

- The flow is due to the influence of the pulsatile pressure gradient and uniform magnetic field.

- A pressure gradient is applied in the $\mathrm{x}$ - direction and a uniform magnetic field is applied in positive $\mathrm{y}$-direction.

- The dust particles are assumed to be spherical in shape and uniformly distributed throughout the fluid.

- Initially the conducting and non-conducting dust particles are assumed to be at rest.

- The number density of the dust particles is taken as a constant.

Under these assumptions the geometry of the flow configuration is shown as in Fig.1

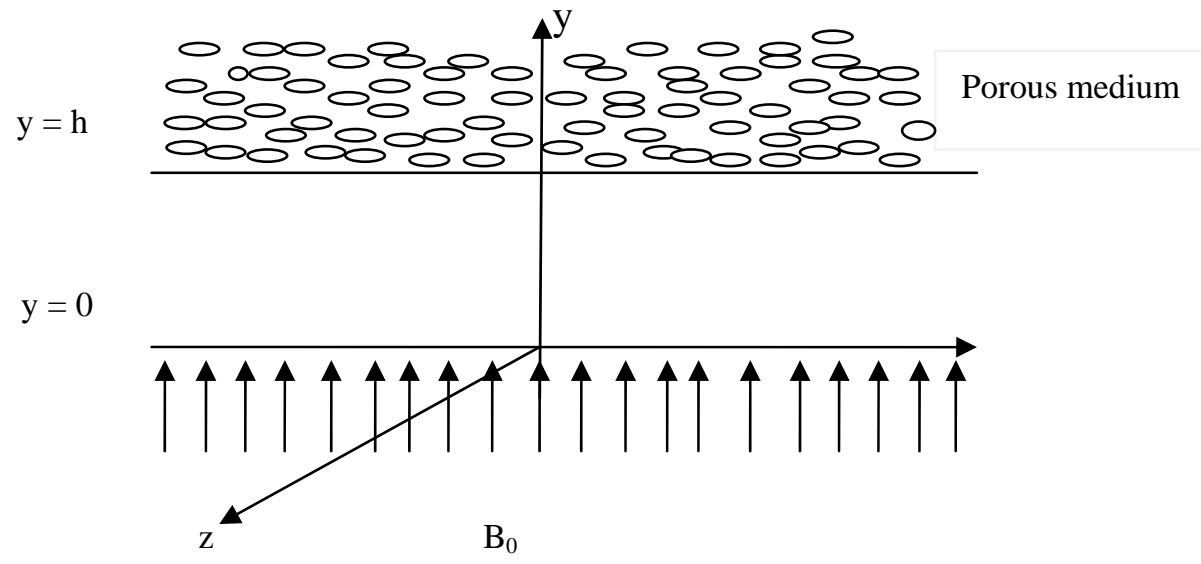

Fig. 1: Geometry of the flow configuration

\section{Equations of Motion}

The governing equations of motion for two phases are given by:

For fluid phase

$$
\begin{gathered}
\nabla \cdot \mathbf{u}=0 \\
\frac{\partial \mathbf{u}}{\partial t}+(\mathbf{u} . \nabla) \mathbf{u}=-\frac{1}{\rho} \nabla p+v \nabla^{2} \mathbf{u}+\frac{K N}{\rho}(\mathbf{v}-\mathbf{u})-\frac{1}{\rho}(\mathbf{J} x \mathbf{B})-\frac{\mu}{k} \mathbf{v}
\end{gathered}
$$

For dust phase

$$
\begin{gathered}
\nabla \cdot \mathbf{v}=0 \\
\frac{\partial \mathbf{v}}{\partial t}+(\mathbf{v} \cdot \nabla) \mathbf{v}=\frac{K}{m}(\mathbf{u}-\mathbf{v})
\end{gathered}
$$

$\mathbf{u}, \rho, p, v$ are respectively velocity vector, density, pressure and kinematic viscosity. $\mathbf{v}, \mathrm{N}, \mathrm{m}$ are respectively velocity vector, number density and mass concentration of dust particles.

\section{Solution of the Problem}

The governing equations from (1) to (4) in the direction(x) of fluid flow are,

\section{For fluid phase}

$$
\frac{\partial u}{\partial t}=-\frac{1}{\rho} \frac{\partial p}{\partial x}+v \frac{\partial^{2} u}{\partial y^{2}}+\frac{K N}{\rho}(v-u)-\frac{\sigma H_{0}^{2}}{\rho} u-\frac{\mu}{k} u
$$




\section{For dust phase}

$$
\frac{\partial v}{\partial t}=\frac{K}{m}(u-v)
$$

Where $u(\mathrm{y}, \mathrm{t})$ and $v(\mathrm{y}, \mathrm{t})$ denote the velocity of the fluid and of dust phase respectively. The initial and boundary conditions on the velocity field are taken as:

\section{Case (i)}

Initial conditions: $u=0, v=0$ at $\mathrm{t} \leq 0$ for all $\mathrm{y}$,

Boundary conditions: $u=a_{1} e^{i \omega_{1} t}+a_{2} e^{-i \omega_{2} t}$ at $\mathrm{y}=0$ for $\mathrm{t}>0 . u=b_{1} e^{i \omega_{1} t}+b_{2} e^{-i \omega_{2} t}$ at $\mathrm{y}=\mathrm{h}$. where $\mathrm{a}_{1}, \mathrm{a}_{2}, \mathrm{~b}_{1}, \mathrm{~b}_{2}$ are complex constants such that u becomes real on plates.

\section{Case (ii)}

Initial conditions: $u=0, v=0$ at $t \leq 0$ for all $\mathrm{y}$.

Boundary conditions: $u=U_{1}$ at $\mathrm{y}=0$ for $t>0, u=V_{l}$ at $\mathrm{y}=\mathrm{h}$.

Here the lower and upper plates start moving with uniform velocities $U_{l}$ and $V_{l}$ respectively.

The pulsatile pressure gradient influenced the flow of fluid and it is of the form,

$$
-\frac{1}{\rho} \frac{\partial p}{\partial x}=A\left[1+\varepsilon e^{i \alpha t}\right]
$$

where $\varepsilon$ is a small quantity, A and $\alpha$ are constants.

Let us consider the following non-dimensional flow variables

$\bar{u}=\frac{u h}{v}, \bar{v}=\frac{v h}{v}, \bar{t}=\frac{v t}{h^{2}}, \bar{x}=\frac{x}{h}, \bar{y}=\frac{y}{h}, \bar{p}=\frac{p h^{2}}{\rho v^{2}}$

Using the above non-dimensional variables in the equations (1) - (4), then one can get the following non-dimensionalzed form of the equations (on dropping the bars as follows):

$$
\begin{aligned}
& \frac{\partial u}{\partial t}=\frac{\partial p}{\partial x}+\frac{\partial^{2} u}{\partial y^{2}}+\frac{f}{\tau_{v}}(v-u)-\left(\sigma^{2}+M^{2}\right) u, \\
& \frac{\partial u}{\partial t}=\frac{1}{\tau_{v}}(u-v)
\end{aligned}
$$

Where $f=\frac{N m}{\rho}$ the mass concentration of dust particles, $\tau_{v}=\frac{m v}{K h^{2}}$ the relaxation time of dust particles, $M=B{ }_{0} h \sqrt{\frac{\sigma}{\mu}}$ Hartmann number. is the porous parameter. The non dimensional form of the initial and boundary conditions on the velocity fields are:

\section{For case (i)}

Initial conditions: $u=0, v=0$ for $t \leq 0$, Boundary conditions: $u=a_{1} e^{i s_{1} t}+a_{2} e^{i s_{1} t}$ at $\mathrm{y}=0$

$u=b_{1} e^{i s_{2} t}+b_{2} e^{-i s_{2} t}$ at $\mathrm{y}=1$. Where $\left(s_{1}, s_{2}\right)=\frac{h^{2}}{v}\left(\omega_{1}, \omega_{2}\right)$ and $\left(\bar{a}_{1}, \bar{a}_{2}, \bar{b}_{1}, \bar{b}_{2}\right)=\frac{h^{2}}{v}\left(a_{1}, a_{2}, b_{1}, b_{2}\right)$

are non-dimensional flow parameters.

\section{For case (ii)}

Initial conditions: $u=0, v=0$ for $t \leq 0$, Boundary conditions: $u=U_{2}$ at $\mathrm{y}=0, u=V_{2}$ at $\mathrm{y}=1$. 
Where $U_{2}=\frac{h}{v} U_{1}$ and $V_{2}=\frac{h}{v} V_{1}$

The non-dimensional pressure gradient as given by

$\frac{\partial p}{\partial x}=A\left[1+\varepsilon e^{i \beta t}\right]$, where $\beta=\frac{\alpha h^{2}}{v}$.

\section{Numerical Solution}

The system of partial differential equations (5) and (6) under initial and boundary conditions for case (i) and case (ii) are solved numerically using finite difference scheme with the help of Matlab software. The purpose of the study is to investigate the effect of porous medium on the unsteady fluid motion within the framework of dusty phase and fluid phase. The unsteady flow within parallel plates under uniform magnetic field has been investigated analytically by Debnath and Ghosh, Mitra and Bhattacharya. The effect of magnetic field on the fluid flow have also been investigated.

\section{Results and Discussion}

In this investigation an unsteady laminar flow of an incompressible viscous fluid with uniform distribution of dust particles between two parallel plates with a uniform magnetic field is considered. Here the flow is studied in the following two cases. (i) Between two parallel plates (ii) impulsive motion of two parallel plates. In both cases the effect of pulsatile pressure gradient is considered. The governing equation for this investigation was non-dimensionalzed and solved numerically using finite difference scheme. In Fig.2 the effect of magnetic parameter on the velocity distributions have been shown for fluid phase. For the purpose choose $\mathrm{t}=0.2$, $\mathrm{dt}=0.5, \mathrm{f}=1.2, \mathrm{~S}=5.0, \mathrm{a}_{1}=2, \mathrm{a}_{2}=3, \mathrm{~b}_{1}=10, \mathrm{~b}_{2}=20, \mathrm{dy}=0.2$, the given system of differential equations is simplified and solved using Matlab software. The magnetic parameter varies from 2.0 to 8.0. It is observed that the velocity for the fluid phase firstly increases then decreases with the distance. With the increase of magnetic parameter the velocity decreases under the influence of porous media.

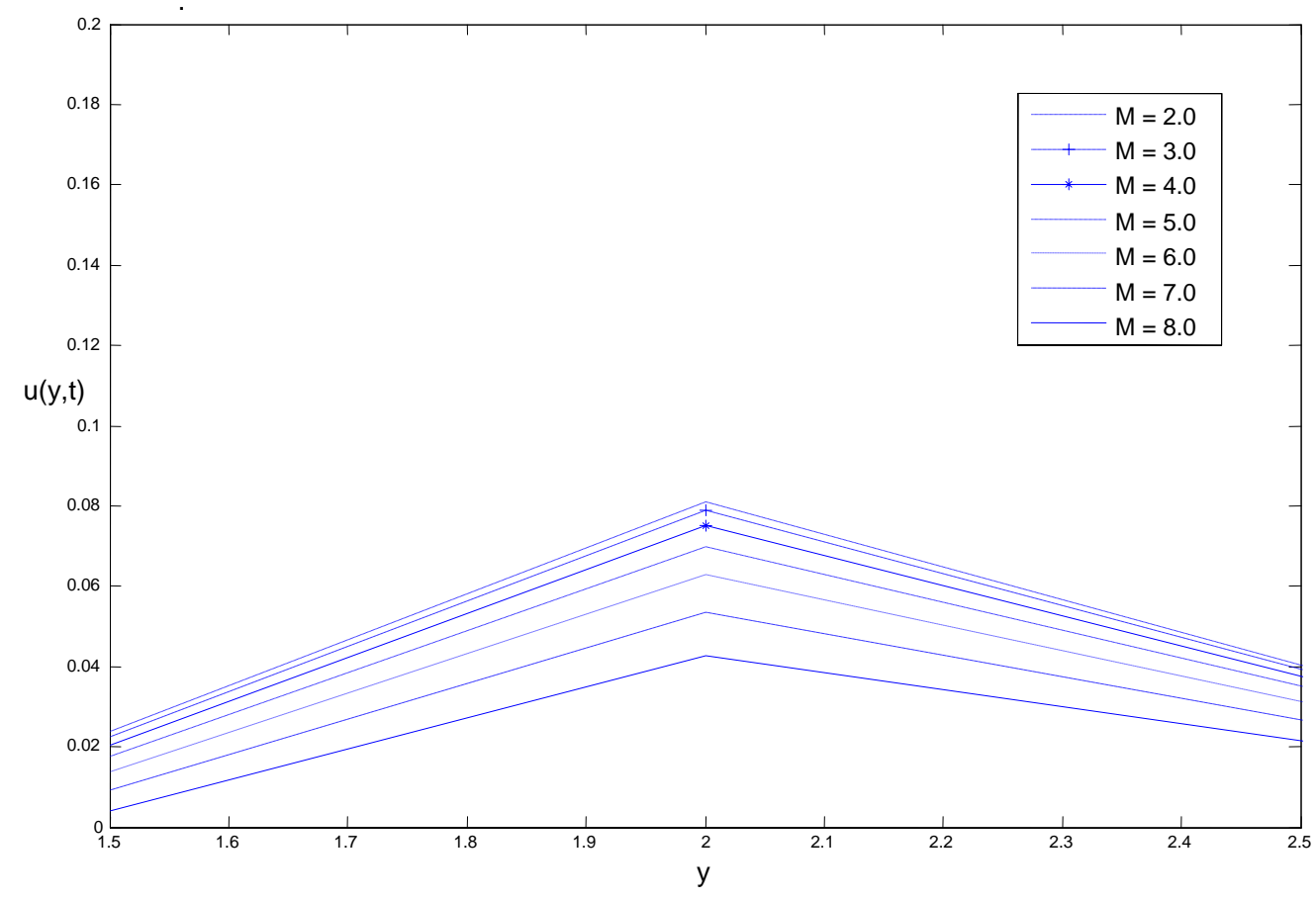

Fig.2 : Influence of magnetic parameter on the velocity distributions for fluid phase

The effect of porous medium on the fluid motion in fluid phase has been shown in Fig. 3 . The graph has been plotted using finite difference scheme. For the purpose choose $\mathrm{t}=0.2, \mathrm{dt}=0.5, \mathrm{f}=1.2, \mathrm{M}=5.0, \mathrm{a}_{1}=2, \mathrm{a}_{2}=3, \mathrm{~b}_{1}=10, \mathrm{~b}_{2}=20, \mathrm{dy}=0.2$, the given system of differential equations is simplified and solved using Matlab software. The porous parameter varies from $\mathrm{S}=2.0$ to 8.0. 
The results reveal that velocity decreases with the increase of porous parameter. It is also observed that velocity increases very slowly from $\mathrm{S}=2.0$ to $\mathrm{S}=4.0$ as distance increases but when the porous parameter increases from $\mathrm{S}=5.0$ to $\mathrm{S}=8.0$ the velocity firstly increases sharply then decreases.

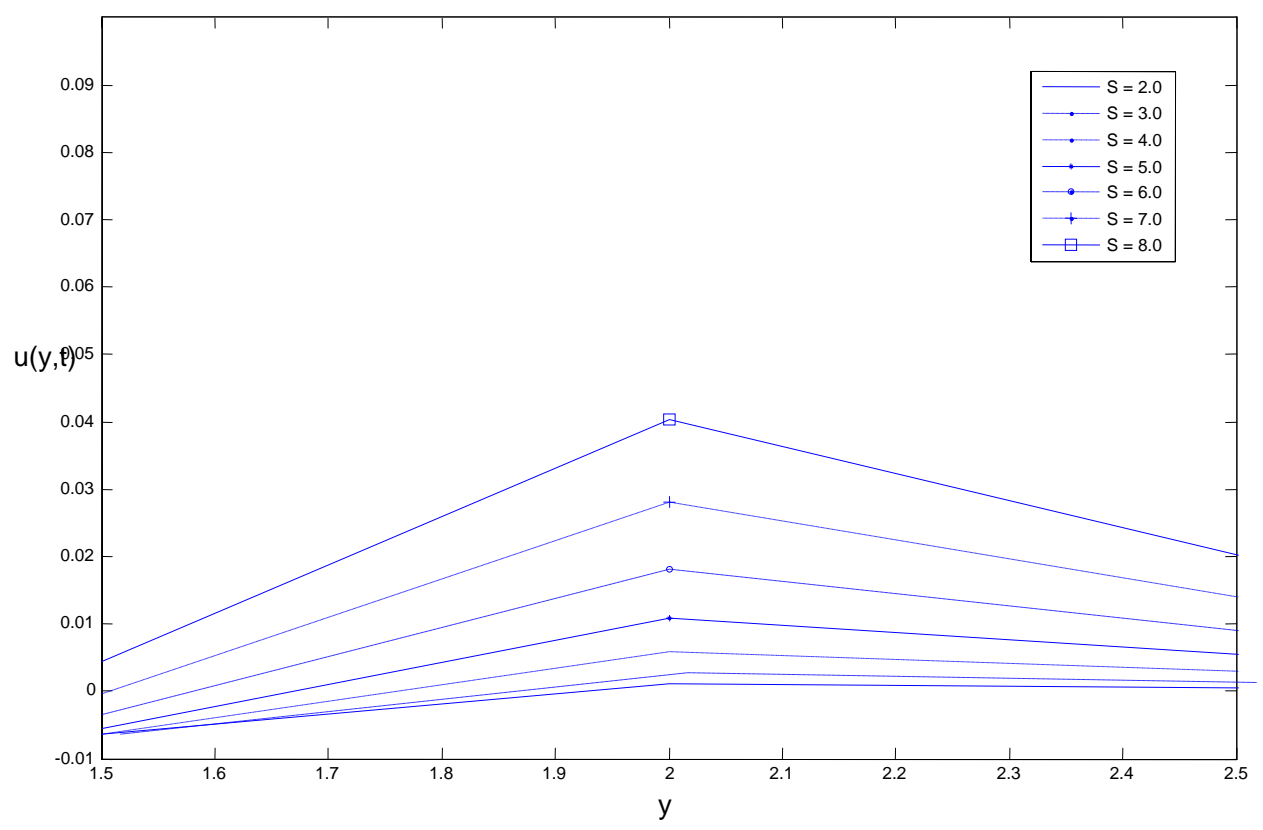

Fig. 3: The effect of porous parameter on the velocity distributions in fluid phase.

The effect of porous medium on the fluid motion in dust phase has been shown in Fig. 4. The graph has been plotted using finite difference scheme. For the purpose choose $\mathrm{t}=0.2, \mathrm{dt}=0.5, \mathrm{f}=1.2, \mathrm{M}=5.0, \mathrm{a}_{1}=2, \mathrm{a}_{2}=3, \mathrm{~b}_{1}=10, \mathrm{~b}_{2}=20$, $\mathrm{dy}=0.2$, the given system of differential equations is simplified and solved using Matlab software. The porous parameter varies from $\mathrm{S}=2.0$ to 9.0.

The graph reveals that velocity firstly increases in the half way of porous medium and then decreases. For small values of the porous parameter the effect on the fluid motion remains negligible.

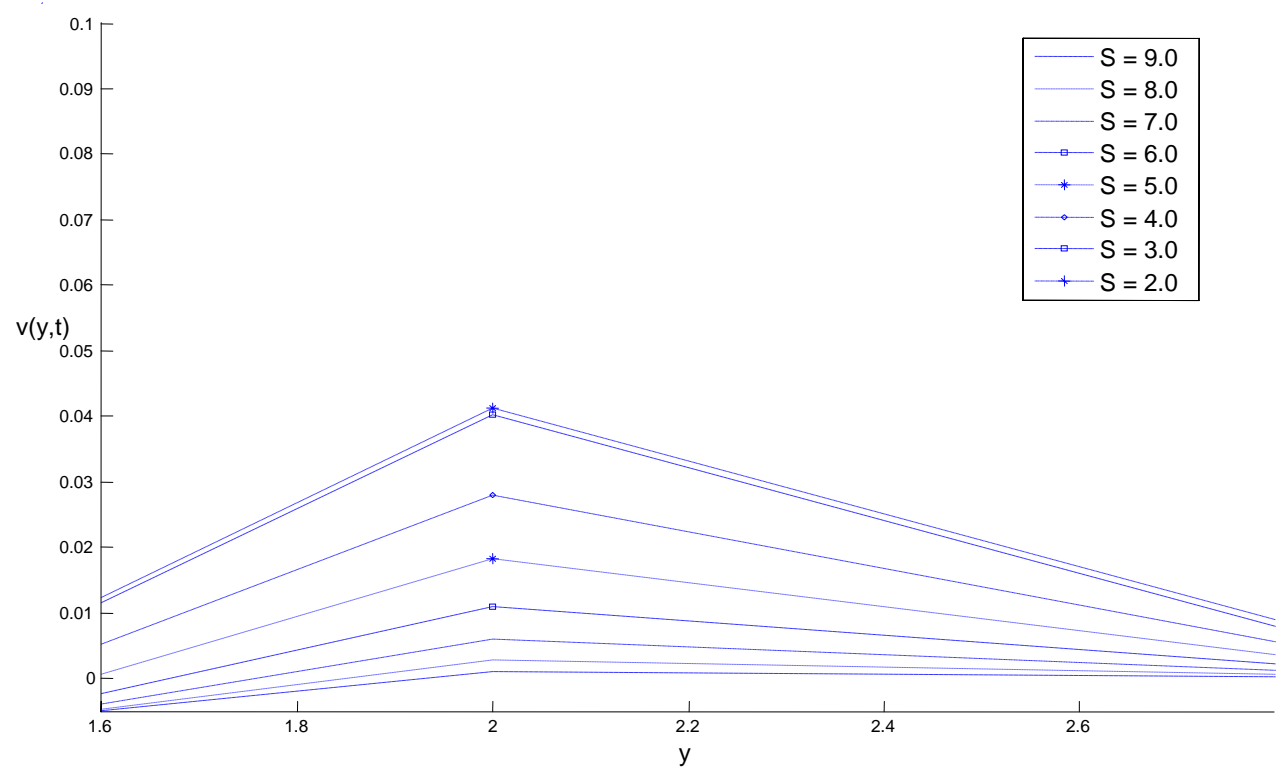

Fig.4 The effect of porous parameter on the velocity of fluid in dust phase

In Fig. 5 the effect of magnetic parameter on the fluid motion has been analyzed. The graph has been plotted using finite difference scheme. For the purpose choose $t=0.2, \mathrm{dt}=0.5, \mathrm{f}=1.2, \mathrm{~S}=5.0, \mathrm{a}_{1}=2, \mathrm{a}_{2}=3, \mathrm{~b}_{1}=10, \mathrm{~b}_{2}=20$, dy $=0.2$, the given system of 
differential equations has been solved by appropriate program and results are deducted through graph. The values of magnetic parameter has been choosed from $M=2.0$ to $M=9.0$. For small values of the magnetic parameter the motion of the fluid remains almost same. But as the value increases the velocity of fluid in dust phase increases sharply. For high values of magnetic parameter there is a point of saturation where the fluid takes almost same affect of magnetic parameter.

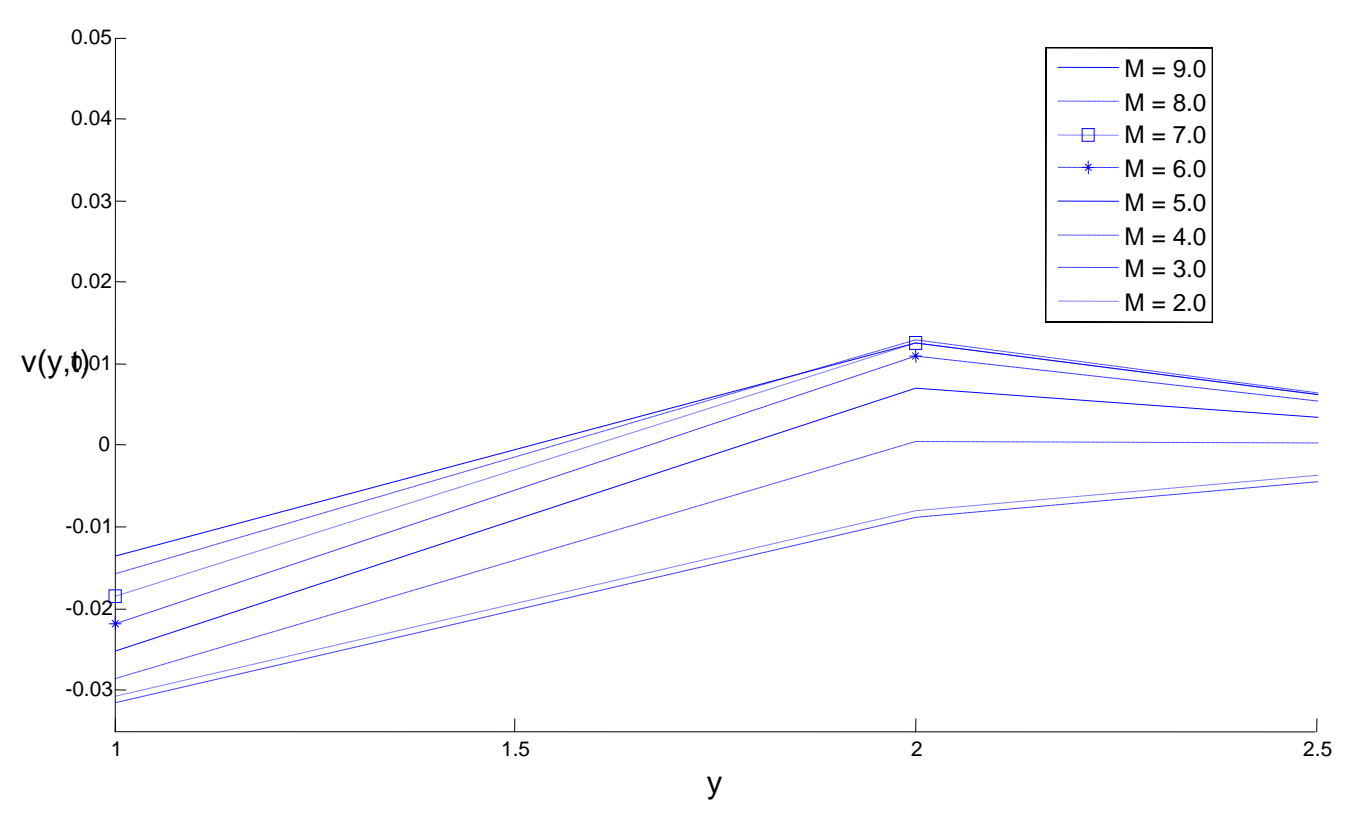

Fig.5: The effect of magnetic parameter on the motion of fluid in dust phase

\section{Acknowledgement}

The author, Parul Saxena, is thankful to the Department of Atomic energy for the financial assistance, Mumbai and to the Department of Mathematics and Astronomy, Lucknow University, Lucknow for research facilities during the work.

\section{References}

Attia H.A. 2006. Unsteady MHD coutte flow and heat transfer of dusty fluid with variable physical properties. Applied Mathematics and Computation, Vol. 177, No. 1, pp. 308-318.

Attia H.A. and Kotb N.A. 1996. MHD flow and heat transfer of dusty fluid between two parallel plates with variable physical properties, Applied Mathematics Modeling, Vol. 26, pp. 863-875.

Chamka A.J. 1998. Unsteady hydromagnetic flow and heat transfer from a non-isothermal stretching sheet immersed in a porous medium. International Communications in Heat and Mass Transfer, Vol. 25, No. 6, pp. 899-906.

Chen C.H. 2004. Combined heat and mass transfer in MHD free convection from a vertical surface with ohmic heating and viscous dissipation. International Journal of Engineering Science, Vol. 42, No. 7, pp. 699-713.

Datta N. , Dalal D.C. and Mishra S.K. 1993. Unsteady heat transfer to pulsatile flow of a dusty viscous incompressible fluid in a channel, International Journal of Heat and Mass Transfer, Vol. 36, No. 7, pp. 1783-1788.

Debnath L. and Ghosh A.K. 1989. On unsteady hydromagnetic flows of a dusty viscous fluid between two oscillating plates, Journal of Applied Mathematics and Simulation, Vol. 2, No. 1, pp. 13-31.

Elbashbeshy E.M.A. and Bazid M.A.A. 2004. Heat transfer over an unsteady stretching surface. Heat and Mass Transfer, Vol. 41, No. 1, pp. 1-4.

Ezzat A.A., Bary E-1 and Morsey M.M. 2010. Space approach to the hydro-magnetic flow of a dusty fluid through a porous medium. Computers and Mathematics with Applications, Vol. 59, No. 8, pp. 2868-2879.

Gireesha H.M., Bagewadi C.S. and Prasannaku B.C. 2009. Pulsatile flow of an unsteady dusty fluid through rectangular channel. Communications in Nonlinear Science and Numerical Simulation, Vol. 14, No. 5, pp. 2103-2110.

Prasad V.R. and Ramacharyulu N.C.P. 1979. Unsteady flow of a dusty incompressible fluid between two parallel plates under an impulsive pressure gradient, Defence Science Journal, Vol. 30, pp. 125-130.

Sreeharireddy P., Nagararajan A. S. and Siviah M. 2009. MHD flow of a dusty viscous conducting liquid between two parallel plates, Journal of Scientific Research, Vol. 1, No. 2, pp. 220-225. 


\section{Biographical notes}

Dr. Parul Saxena received Ph.D. in 2008 from Lucknow University. She is currently working as NBHM Postdoctoral Fellow in the Dept. of Mathematics and Astronomy, Lucknow University, Lucknow. Her research interests include fluid flow through porous media and their applications.

Prof. Manju Agarwal, Ph.D. in Applied Mathematics, was Professor in charge, Library, Institute of Engineering and Technology from 1985 -1988. She worked as an editorial Secretary of Ganita from 1993-2008. Presently working as Head of the Department, Department of Mathematics and Astronomy, Lucknow University, Lucknow. Her research interests are mathematical modeling, environmental pollution, mathematical ecology and fluid dynamics.

Received February 2013

Accepted August 2013

Final acceptance in revised form September 2013 